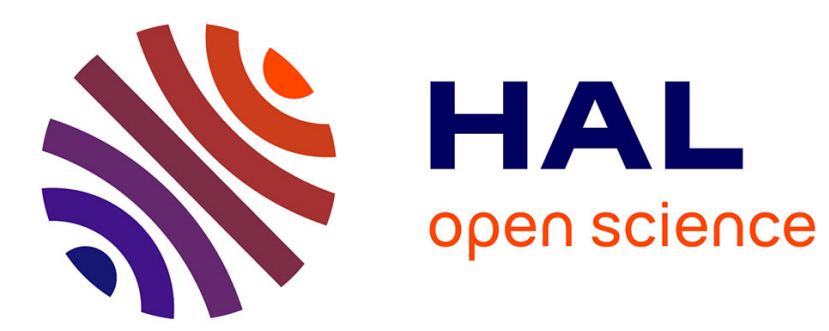

\title{
Contribution à l'étude des pics d'écrouissage à très basse température
}

\author{
P. Mazot, J. Petit
}

\section{To cite this version:}

P. Mazot, J. Petit. Contribution à l'étude des pics d'écrouissage à très basse température. Revue de Physique Appliquée, 1976, 11 (5), pp.597-601. 10.1051/rphysap:01976001105059700 . jpa-00244094

\section{HAL Id: jpa-00244094 https://hal.science/jpa-00244094}

Submitted on 1 Jan 1976

HAL is a multi-disciplinary open access archive for the deposit and dissemination of scientific research documents, whether they are published or not. The documents may come from teaching and research institutions in France or abroad, or from public or private research centers.
L'archive ouverte pluridisciplinaire HAL, est destinée au dépôt et à la diffusion de documents scientifiques de niveau recherche, publiés ou non, émanant des établissements d'enseignement et de recherche français ou étrangers, des laboratoires publics ou privés. 
Classification

Physics Abstracts

7.240

\title{
CONTRIBUTION A L'ÉTUDE DES PICS D'ÉCROUISSAGE A TRÈS BASSE TEMPÉRATURE
}

\author{
P. MAZOT et J. PETIT \\ Laboratoire de Mécanique et de Physique des matériaux, E. N. S. M. A., Poitiers, France
}

(Reçu le 22 mars 1976, révisé le 20 mai 1976, accepté le 8 juin 1976)

\begin{abstract}
Résumé. - Les récents travaux sur les pics de frottement intérieur dus à l'écrouissage dans les métaux de structure cubique centrée ont montré la nécessité de tracer les spectres d'amortissement à partir des très basses températures. Nous avons donc réalisé un appareil qui permette de tracer ces spectres de 10 à $300 \mathrm{~K}$ sur des éprouvettes sollicitées en vibration longitudinale (entre $10 \mathrm{et} 100 \mathrm{kHz}$ ). Quelques premiers résultats sur les pics $\mathrm{P}_{\boldsymbol{\delta}}$ du Tantale, du Niobium et du Molybdène sont alors exposés.

Abstract. - Previous works on internal friction peaks induced by coldwork in bcc metals had shown that it was interesting to determine their relaxation spectra in the very low temperature range. New equipment has been realized, allowing internal friction measurements from $10 \mathrm{~K}$ to $400 \mathrm{~K}$, in longitudinal vibrations from 10 to $100 \mathrm{kHz}$. This paper presents this equipment and the first results related to the relaxation peaks $\mathbf{P}_{\boldsymbol{\delta}}$ in Tantalum, Niobium and Molybdenum.
\end{abstract}

Introduction. - Depuis de nombreuses années un consensus général s'était établi pour identifier les pics $P_{\alpha}$, dans les métaux de structure cubique centrée, à des pics de type Bordoni, de même nature que ceux étudiés sur les métaux de structure cubique face centrée par Bordoni [1], Niblett [2, 3]... L'analyse théorique des phénomènes de relaxation associés à ces pics a donné lieu à diverses interprétations $[4,5,6,7,8]$.

Cependant dans les années 68-69 Mazzolaï, Nuovo et Cannelli $[9,10]$ émettaient de nouvelles hypothèses sur l'interprétation des pics d'écrouissage pour les métaux de structure cubique centrée : d'une part ils mettaient en évidence dans le Tantale, le Niobium et le Vanadium un pic $P_{\delta}$ situé à très basse température (dans la zone 10-40 K) ; d'autre part, sur la base de leurs expériences révélant le rôle de l'hydrogène sur $P_{\alpha}$ et $P_{\delta}$, ils affirmaient que $P_{\delta}$ était le véritable pic de Bordoni et que $\mathrm{P}_{\alpha}$ était en fait le pic de Koster associé à l'hydrogène, reprenant sur ce dernier point une interprétation beaucoup plus ancienne de Bruner [11].

En fonction de ces nouvelles hypothèses et de travaux antérieurs dans notre laboratoire sur ce thème de recherche $[8,12,13,14]$ nous avons décidé de mettre au point un appareil qui nous permette d'obtenir les spectres de frottement interne à partir de la température de l'hélium liquide.

1. L'appareil de mesure du frottement interne à très basse température. - La figure 1 montre le schéma

(*) E.R.A. au C.N.R.S. $n^{\circ} 123$.

REVUE de PHYSIQUe APPLIQUÉE. - T. 11, No 5, SEPTEMBRE 1976

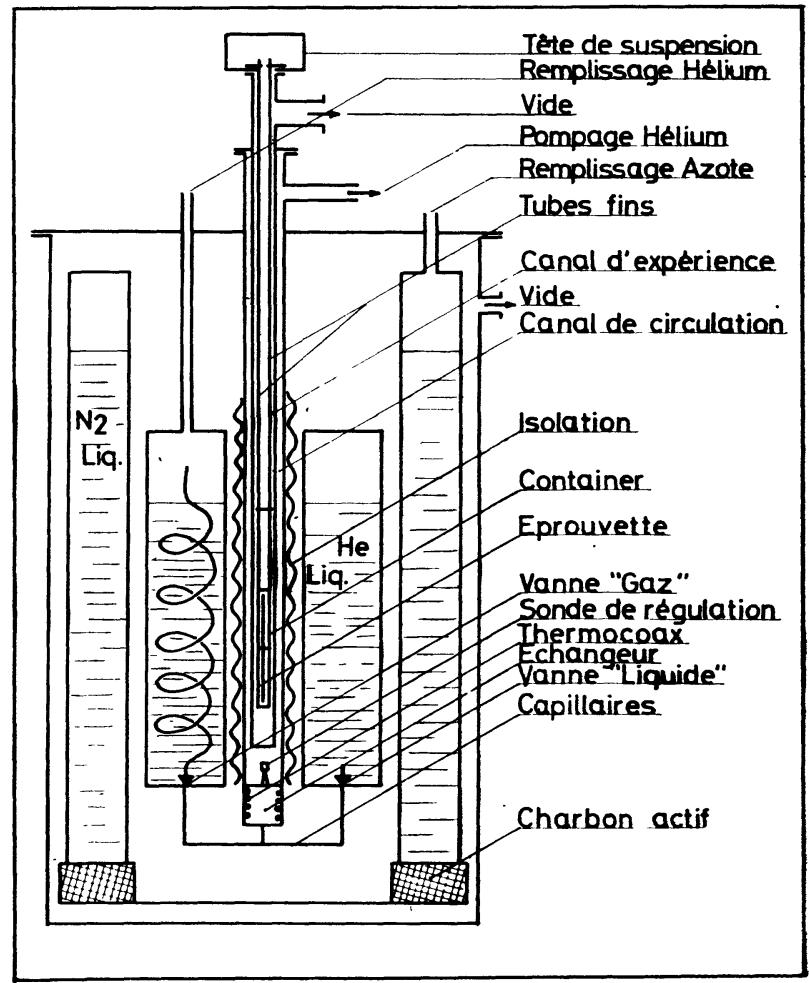

Fig. 1. - Schéma du cryostat.

général de l'appareil ; il s'agit d'un cryostat à hélium liquide, avec garde d'azote liquide et refroidissement par circulation d'hélium, conçu par la Société MERIC. Le canal d'expérience dans lequel il est possible de faire 
un vide primaire ou secondaire, est plongé dans un canal de circulation d'hélium. Le réservoir d'hélium liquide est relié par deux vannes pointeaux (gaz et liquide) et par deux capillaires à un échangeur situé au pied du canal de circulation et dans lequel est enserré un thermocoax de réchauffage. Les variations de température sont obtenues en agissant sur le débit de la pompe de circulation de l'hélium d'une part, sur l'intensité envoyée dans le thermocoax de réchauffage d'autre part. Une sonde As-Ga placée au-dessus de l'échangeur permet une régulation de température par action proportionnelle ou dérivée sur l'intensité de réchauffage.

L'éprouvette et son container sont descendus dans le canal central, le tout étant suspendu par des tubes inox minces (1/10 mm épaisseur) et relié par ailleurs à la tête de suspension supérieure par : un fil d'excitation, un fil de lecture, deux fils d'alimentation d'une sonde As-Ga fixée sur le container, deux fils d'un thermocouple $\mathrm{AuFe} / \mathrm{NiCr}$ fixé soit sur le container, soit sur une éprouvette témoin pour les étalonnages ; tous ces fils étant aussi fins que possible. Enfin le container est mis en contact avec le tube central par des frotteurs en cuivre. Tout ceci permet de débuter le tracé d'un spectre de frottement interne à partir de $5 \mathrm{~K}$, avec une vitesse de remontée relativement forte au départ (de l'ordre de $60 \% / \mathrm{h}$ ) mais qui décroît très vite à partir de $20 \mathrm{~K}$ et que l'on peut alors stabiliser à $30^{\circ} / \mathrm{h}$ en jouant sur l'intensité de réchauffage.

Actuellement le container introduit dans le canal central d'expérience (Fig. 2) permet la sollicitation d'éprouvettes cylindriques en vibrations longitudinales ; l'excitation et la lecture se font par une méthode capacitive [15], ceci expliquant la nécessité d'opérer sous vide. Les éprouvettes sont de deux sortes : des éprouvettes cylindriques de diamètre 3 à $6,5 \mathrm{~mm}$ et de longueur 55 à $80 \mathrm{~mm}$ que l'on écrouit en compression, des éprouvettes cylindriques de diamètre $3 \mathrm{~mm}$ avec des têtes cylindriques de $6 \mathrm{~mm}$ de diamètre que l'on écrouit alors par traction.

On peut faire varier la fréquence de résonnance en faisant varier la longueur ou les dimensions respectives tête/éprouvettes. Cependant le plus intéressant est de tracer simultanément, lors d'une même montée en température et sur la même éprouvette, les spectres de frottement interne pour la fréquence de résonnance fondamentale et pour la troisième harmonique ; ceci permet d'écarter le problème de la reproductibilité de la montée en température et de déterminer avec une meilleure précision le déplacement en température des pics activés thermiquement, d'autant qu'à ces températures très basses ce déplacement est très faible.

Les phénomènes de relaxation à très basse température se traduisent généralement par des pics d'amortissement de faible amplitude (de l'ordre de quelques $10^{-5}$ ) ce qui nécessite une bonne résolution de la méthode de mesure ; c'est pourquoi nous avons choisi la méthode des barreaux résonnants qui dans nos conditions d'application donne une résolution de l'ordre de $10^{-6}$. Cependant il est parfaitement envisa-
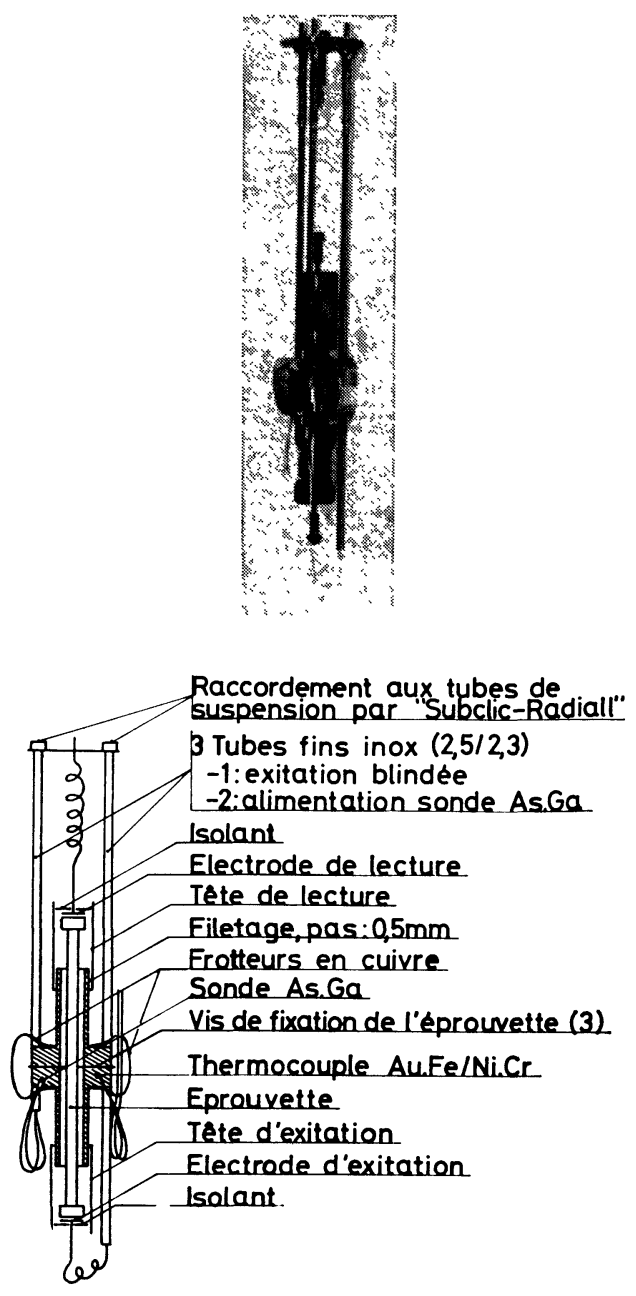

Fig. 2. - Schéma du container pour sollicitation en vibration longitudinale.

geable de descendre dans le cryostat un autre type de container dans lequel des éprouvettes seraient sollicitées en flexion encastrée, flexion libre ou torsion.

2. Quelques premiers résultats. - Après le stade de la mise au point et des étalonnages nous avons réalisé sur cet appareil quelques expériences qui ont porté d'une part sur les métaux de structure hexagonale, Titane et Zirconium et qui font l'objet d'une autre publication [16] ; d'autre part sur les métaux de structure cubique centrée, Niobium, Tantale et Molybdène ; ce sont ces derniers résultats que nous allons exposer ici. Les teneurs en impuretés des métaux utilisés sont indiquées dans le tableau I.

\begin{tabular}{|c|c|c|c|c|c|c|c|c|c|c|c|c|c|c|c|}
\hline & & & eurs & en & impu & $\begin{array}{l}\text { TAE } \\
\text { uretés }\end{array}$ & $\begin{array}{l}B L \\
s \text { de }\end{array}$ & $\begin{array}{l}E A l \\
\mathrm{~s} \mathrm{~m}\end{array}$ & $\begin{array}{l}\mathrm{JI} \\
\text { étau }\end{array}$ & $x$ utrl & lisés & & & & \\
\hline & C & 0 & $\mathbf{N}$ & $\mathrm{H}$ & $\mathrm{Fe}$ & $\mathrm{Ta}$ & Mo & $\mathrm{Nb}$ & $w$ & $\mathrm{Cr}$ & $\mathrm{Al}$ & $\mathrm{Si}$ & $\mathrm{Cu}$ & $\mathrm{Ni}$ & $\mathrm{Ti}$ \\
\hline $\mathrm{Ta}$ & 25 & 50 & 20 & 5 & 10 & & $<10$ & 60 & 70 & $<2$ & $<10$ & $<10$ & $<2$ & $<1$ & $<5$ \\
\hline $\begin{array}{c}\mathrm{Nb} \\
K . L\end{array}$ & 30 & 20 & 90 & $<10$ & 20 & 500 & - & & $<10$ & - & - & $<10$ & - & - & $<10$ \\
\hline $\mathrm{Mo}_{J M}$ & 10 & 14 & 12 & 1 & 40 & - & & - & - & 30 & - & 3 & 3 & 20 & - \\
\hline
\end{tabular}


2.1 Essais Sur LE TANTAle. - Les figures 3 et 4 montrent que nos résultats confirment ceux déjà obtenus par Mazzolai et Nuovo [9]: on observe, autour de $30 \mathrm{~K}$ pour le domaine de fréquence utilisé, un pic $P_{\delta}$ d'amplitude assez faible pour un écrouissage déjà élevé ; l'allure du spectre (dédoublement du sommet du pic et largeur à mi-hauteur) montre qu'il ne

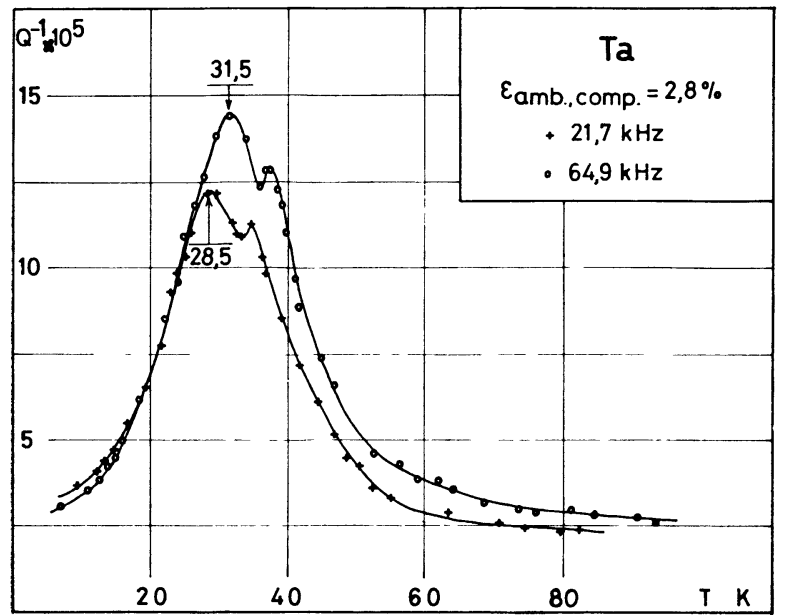

Fig. 3. - Le pic $P_{\delta}$ du Tantale, déplacement en fonction de la fréquence.

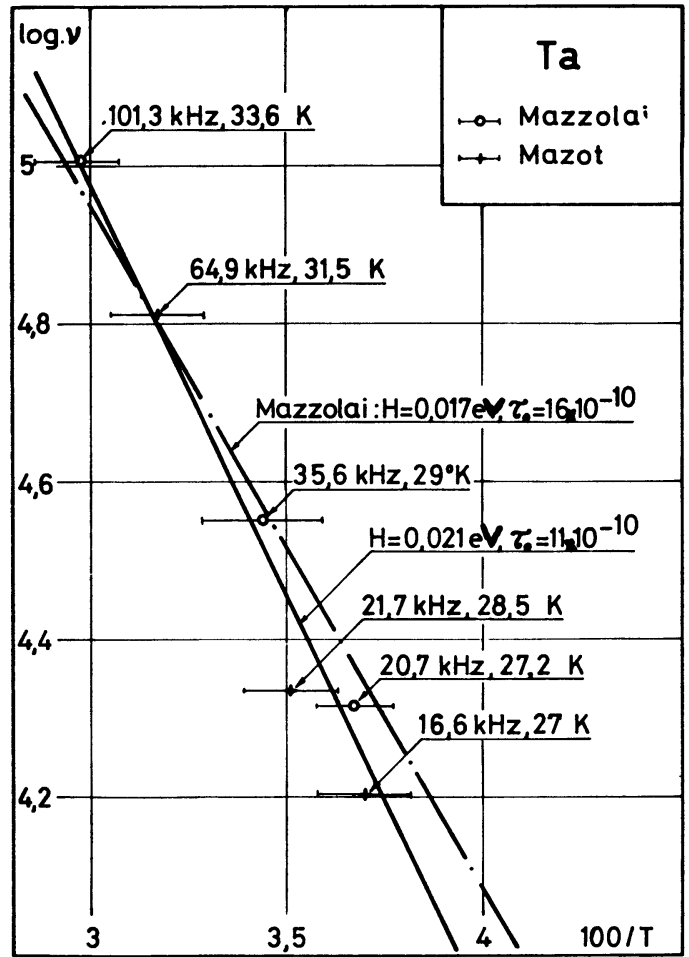

Fig. 4. - Variation de la température du sommet du pic $P_{\delta}$ du Tantale en fonction de la fréquence.

s'agit pas d'un pic simple de Debye ; enfin en tenant compte de nos points expérimentaux et de ceux de Mazzolai et Nuovo nous avons redéterminé les caractéristiques du pic :

$$
H_{\delta}=0,021 \mathrm{eV} \pm 0,005, \quad \tau_{0 \delta}=11 \times 10^{-10 \pm 1}
$$

qui, bien que légèrement différentes, restent du même ordre de grandeur que celles données par les auteurs précédents. En utilisant cette valeur de $H_{\delta}$ on trouve d'après les tables données par Novick et Berry [17] un facteur d'élargissement du pic $P_{\delta}$ :

$$
\beta_{\delta}=2,9 \pm 0,7 \text {. }
$$

On peut aussi observer sur cet essai le très faible déplacement du pic malgré la faible valeur de $H_{\delta}$; on voit alors l'intérêt de tracer les deux spectres (fréquence fondamentale et troisième harmonique) lors d'une même remontée en température afin d'évaluer $\Delta T$ avec le maximum de précision; malgré cela l'erreur sur $H_{\delta}$ et $\tau_{0 \delta}$ reste importante.

2.2 ESSAIS SUR LE NiobIUM. - Sur ce métal les résultats antérieurs essentiels ont été donnés par Mazzolai et Nuovo [9] qui décèlent un pic $P_{\delta}$ vers $34 \mathrm{~K}$ pour une fréquence de $41,5 \mathrm{kHz}$ puis par Wert, Thompson et Buck [18] qui, pour leur part, observent ce pic vers $40 \mathrm{~K}$ pour une fréquence de $13 \mathrm{kHz}$. Mais, à notre connaissance, aucune valeur n'a été donnée sur les caractéristiques associées à ce pic : énergie d'activation $H_{\delta}$ et temps limite $\tau_{0 \delta}$.

Nos résultats essentiels sur ce métal sont réunis dans les figures 5,6 et 7 . La figure 5 montre l'évolution du pic $P_{\delta}$ avec l'écrouissage : on observe une croissance rapide de la hauteur du pic, $Q^{-1} \max$, en fonction du taux de déformation, sans qu'une saturation commence à apparaître pour une déformation allant jusqu'à $5 \%$; ceci étant, ces valeurs de $Q^{-1}$ max restent très faibles (de l'ordre de quelques $10^{-5}$ ) comparées à celles obte-

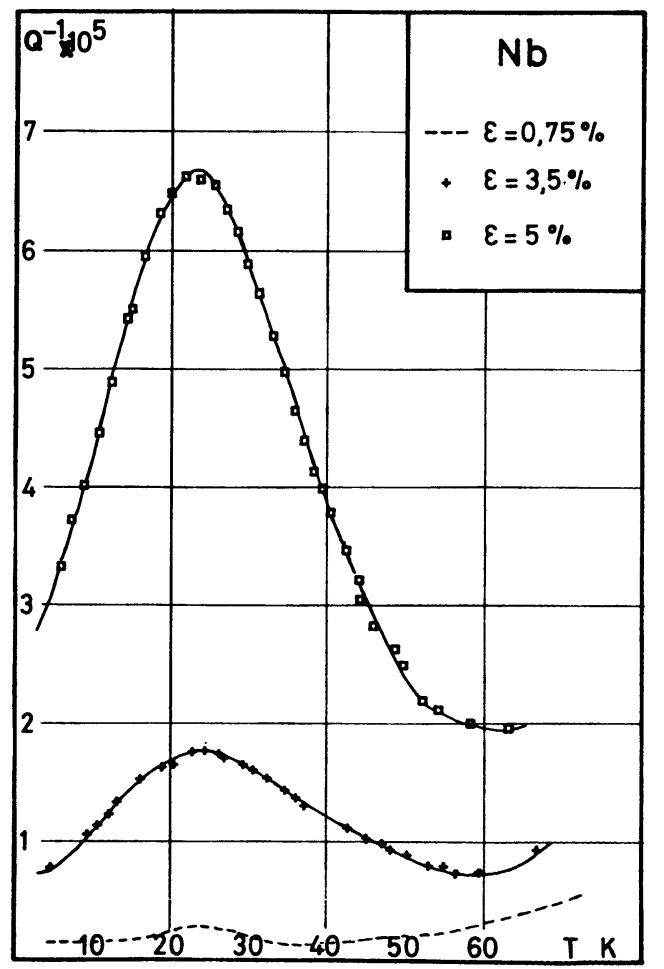

Fig. 5. - Le pic $\mathrm{P}_{\delta}$ du Niobium, évolution en fonction du taux d'écrouissage. 


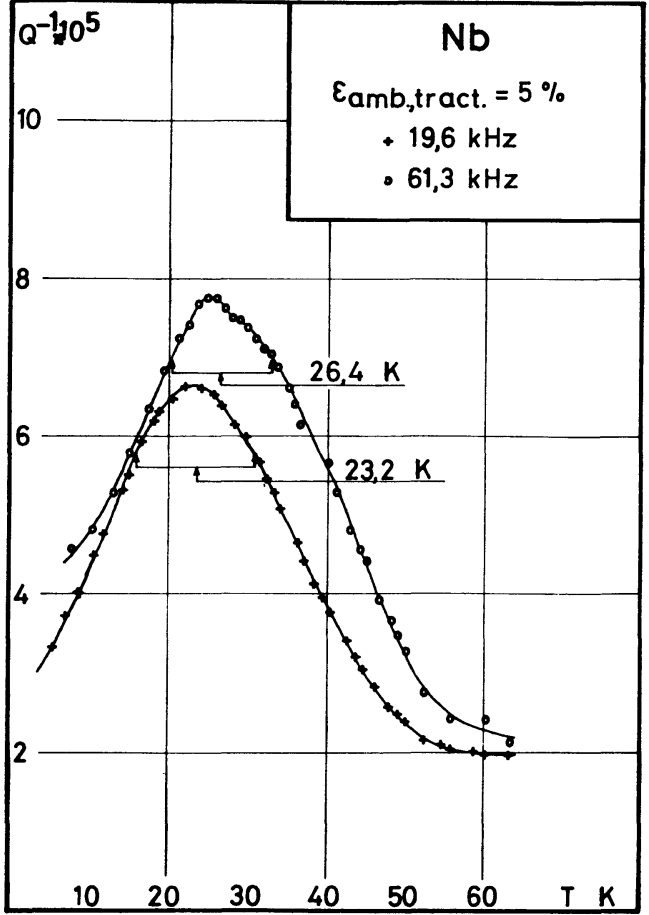

Fig. 6. - Déplacement du pic $\mathbf{P}_{\delta}$ du Niobium en fonction de la fréquence.

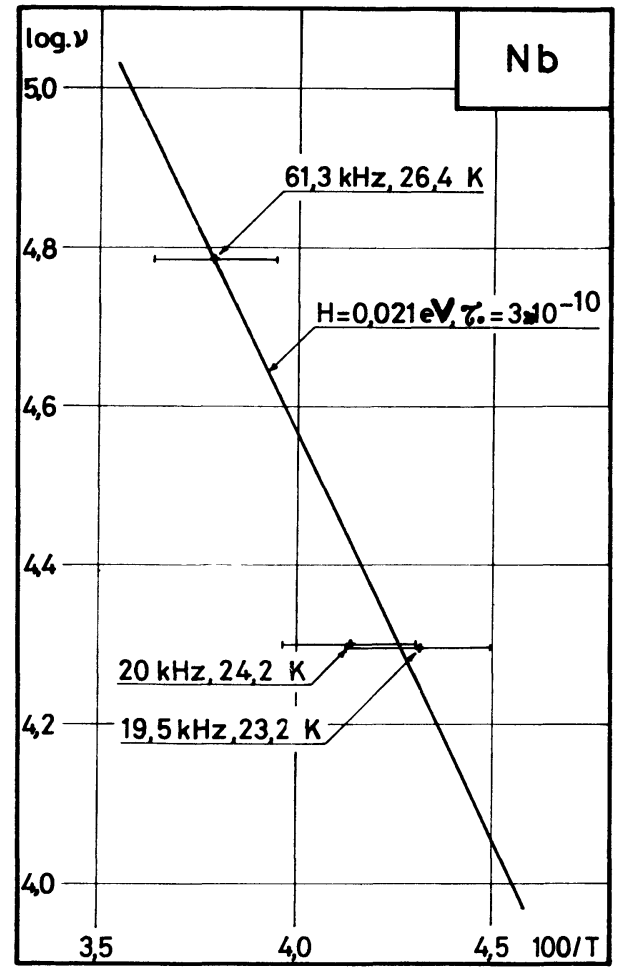

FIG. 7. - Variation de la température du sommet du pic $\mathbf{P}_{\boldsymbol{\delta}}$ du Niobium en fonction de la fréquence.

nues pour $\mathrm{P}_{\alpha}$ à écrouissage identique (pour ce dernier pic $Q^{-1}$ max dépasse $10^{-3}$ dès $3 \%$ d'écrouissage). Par ailleurs le spectre obtenu pour une éprouvette écrouie de $0,75 \%$ seulement montre la sensibilité de la méthode des barreaux résonnants qui permet de déceler un pic dont l'amplitude est de l'ordre de $10^{-6}$ déduction faite $\mathrm{du}$ fond continu.

Sur la figure 6 nous avons tracé les deux spectres obtenus pour la fréquence fondamentale d'une part et l'harmonique 3 d'autre part, lors d'une même montée en température. Le déplacement en température, $\Delta T$, du pic donne, comme cela est reporté sur la figure 7, une énergie d'activation $H_{\delta}=0,021 \mathrm{eV} \pm 0,005 \mathrm{eV}$ et un temps limite

$$
\tau_{0 \delta}=3 \times 10^{-10 \pm 1} ;
$$

ces valeurs sont bien du même ordre que celles obtenues pour le pic $\mathbf{P}_{\boldsymbol{\delta}}$ du Tantale.

Notons encore que, comme pour le Tantale, le pic $\mathbf{P}_{\boldsymbol{\delta}} \mathrm{du}$ Niobium n'est pas un pic simple de Debye ; la forme du sommet du pic (Fig. 6) le montre clairement.

2.3 EsSAIS SUR LE Molybdène. - Sur ce métal nous ne connaissons aucune expérience précise à très basse température révélant un pic $P_{\delta}$ du même type que celui observé sur le Tantale, le Niobium ou le Vanadium [10] ; Rieu dans une récente publication [19] met en évidence sur des monocristaux de Molybdène, un dédoublement du pic $P_{\alpha}$ en deux pics qu'il dénomme « $\mathbf{P}_{\delta}$ " et $~ " \mathrm{P}_{\alpha}$ "; ces deux pics, situés respectivement à $113 \mathrm{~K}$ et $173 \mathrm{~K}$ pour une fréquence de $60 \mathrm{kHz}$, sont tous les deux d'amplitude très élevée $\left(Q^{-1} \max\right.$ supérieur à $7 \times 10^{-3}$ pour un écrouissage de $1 \%$ ); Rieu évalue leur énergie d'activation respective à $0,11 \mathrm{eV}$ et $0,3 \mathrm{eV}$.

Nos propres résultats sur le Molybdène nous amènent à des conclusions différentes de celles de Rieu. La figure 8 montre qu'à très basse température (en dessous de $100 \mathrm{~K}$ ) on observe bien le pied du pic « $\mathrm{P}_{\delta}$ » de Rieu, mais qu'un tracé très précis du spectre permet de déceler deux pics d'amplitude très faible (quelques $10^{-5}$ ) par rapport à celle des pics de Rieu, et qui sont donc presque noyés dans le pied du pic $« \mathrm{P}_{\delta} »$. Dans ces conditions nous pensons que le pic $« \mathrm{P}_{\delta} »$ trouvé par Rieu est du même type que $" \mathrm{P}_{\alpha}$ " (même comporte-

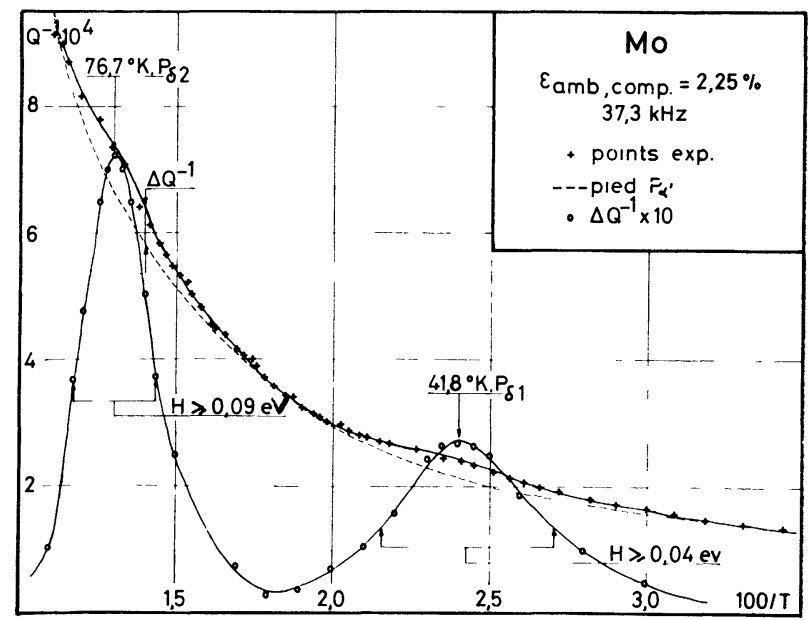

Fig. 8. - Spectre de frottement interne du Molybdène entre 30 et $100 \mathrm{~K}$. 
ment, même ordre de grandeur de l'amplitude...) et qu'il est plus logique de le noter $\mathbf{P}_{\alpha^{\prime}}$; par contre les deux pics que nos essais ont mis en évidence (Fig. 8) ont une amplitude comparable à celle des pics $\mathrm{P}_{\delta} \mathrm{du}$ Niobium et du Tantale et se situent dans la même zone de température, c'est pourquoi nous les appellerons respectivement $\mathbf{P}_{\delta_{1}}$ et $\mathbf{P}_{\delta_{2}}$.

Nos résultats, malgré les difficultés de détermination dues à la présence proche de $P_{\alpha^{\prime}}$ permettent de situer ces pics $P_{\delta_{1}}$ et $P_{\delta_{2}}$ respectivement vers $42 \mathrm{~K}$ et $77 \mathrm{~K}$ pour une fréquence de l'ordre de $37 \mathrm{kHz}$. A partir de la largeur approximative des pics (Fig. 8) et en supposant qu'il s'agisse de pics de Debye, on peut faire une première évaluation de l'énergie d'activation associée à ces pics :

$$
H_{\delta_{1}} \simeq 0,04 \mathrm{eV}
$$

et

$$
H_{\delta_{2}} \simeq 0,09 \mathrm{eV}
$$

On en déduit :

$$
\begin{aligned}
& \tau_{0 \delta_{1}} \simeq 7 \times 10^{-11} \\
& \tau_{0 \delta_{2}} \simeq 0,6 \times 10^{-11} .
\end{aligned}
$$

Ces dernières valeurs n'ont qu'une valeur indicative et devront être précisées par de nouvelles expériences.

\section{Bibliographie}

[1] Bordon, P. G., Nuovo Cimento 17 (1960) 43.

[2] Niblett, D. H. et Wilks, J., Phil. Mag. 1 (1956) 415.

[3] NibletT, D. H., Phys. Acoust., Vol. III, chap. 3, part. A (1966).

[4] SeEger, A., Phil. Mag. 1 (1956) 651.

[5] Seeger, A. et Schiller, P., Acta Met. 10 (1962) 348.

[6] Brailsford, D., Phys. Rev. 122 (1961) 778.

[7] Escaig, B., Script. Met. 5 (1971) 199.

[8] RIEU, G., Thèse Poitiers (1970).

[9] Mazzolai, F. M. et Nuovo, M., Solid State Commun. 7 (1968) 103.

[10] Cannelli, G. et Mazzolai, F. M., J. Phys. Chem. Solids 31 (1970) 1913.

[11] Bruner, L. J., Phys. Rev. 118 (1960) 399.

[12] De Fouquet, J., Boch, Ph., Petit J. et Rieu G., J. Phys. Chem. Solids 31 (1970) 1901.
[13] Rieu, G. et De Fouguet J., J. Physique Colloq. 32 (1971) C 2-221.

[14] Ferron, G., Riviere, A., Quintard, M., Petit, J. et DE FouQuet, J., Script. Met. 9 (1975) 697.

[15] Boch, P., Mallet, J. M. et De Fouquet, J., Mem. Sci. Rev. Met. 6 (1967) 507.

[16] Petit, J., Mazot, P. et De Fouquet, J., Communication to "Second European conference on internal friction" Rome sept. 75, à paraître dans Nuovo Cimento.

[17] Nowick, A. S. et BERRY, B. S., IBM Journal 5 (1961) 257.

[18] Wert, C., Thompson, D. O. et Buck, O., J. Phys. Chem. Solids 31 (1970) 1793.

[19] RIEU, G., à paraître dans Script Met., Communication to «Second European conference on Internal friction » Rome sept. 75. 